\title{
When Items Become Victims: Brand Memory in Violent and Nonviolent Games
}

\author{
André Melzer, Brad J. Bushman, and Ulrich G. Hofmann \\ A. Melzer, Université du Luxembourg, Luxembourg \\ andre.melzer@uni.lu \\ B. J. Bushman, University of Michigan, Ann Arbor, USA, and VY University Amsterdam, \\ The Netherlands \\ bbushman@umich. edu \\ U. G. Hofmann, University of Luebeck, Germany \\ hofmann@imis.uni-luebeck.de
}

\begin{abstract}
This paper introduces the AdRacer system for multifaceted testing and in-depth analyses of game effects and in-game advertising efficiency. AdRacer combines an immersive driving simulator, 3D game environment, recording of players' gaze directions, and application of memory tests. A pilot study tested the effects of game violence on memory for brands shown as billboard ads in a racing game. In contrast to findings with TV violence, game violence did not impede brand memory. Memory results were also not mediated by visual attention during encoding. Compared to a matching nonviolent version, playing a violent game resulted in superior brand retrieval, yet participants showed fewer and shorter eye fixations on the billboard ads. Hence, caution seems to be recommended in transferring standard results from the "passive" TV medium to the interactive game medium.
\end{abstract}

Keywords: Game violence, in-game advertising, memory, media effects.

\section{Introduction}

Violent and nonviolent computer and video games have been analyzed with respect to their emotional, behavioral, and cognitive effects (e.g., Anderson \& Bushman, 2001). To our knowledge, however, the effects of video game violence on memory for advertised brands have not yet been tested. The present paper introduces $A d$ Racer, a novel testing system for the study of game effects. AdRacer warrants high levels of experimental control in a realistic and immersive 3D game environment. The system was tested in a pilot study on the effects of playing a violent or nonviolent racing game on memory for brands. Brand information was provided as billboard ads, thus matching what is found on real race tracks. This so-called in-game advertising denotes the contextual placement of brands within games and is currently becoming increasingly important for advertisers (section 2). Following a detailed description of the AdRacer system (section 3), we will discuss the importance of analyzing effects of game violence on brand memory and report results from the pilot study (section 4). 


\section{In-Game Advertising}

In-game advertising has become a major topic because of people's dramatic changes in (entertainment) media behavior. By middle childhood, for example, playing computer and video games has become one of the favorite leisure-time activities (cf. von Salisch, Oppl, \& Kristen, 2006), thus rivaling TV as the former anchor medium for entertainment. In addition, media diversification describes peoples' tendency to using various other sources of media entertainment such as mobile phones, PDAs, and, video games. The ever-accelerating economic importance of in-game advertising is also reflected by the numbers. In 2006, companies spent $\$ 77.7$ million for in-game advertising. In 2007, the budget more than doubled to $\$ 182.7$ million, whereas in 2011 it is projected that $\$ 971.3$ million will be spent on in-game advertising (Yankee Group ${ }^{1}$ ).

Advertisers' step into games is promising because video games grant foolproof access to the coveted target group of young adults who are known to be avid gamers. Young adults also have more expendable income than other groups, and have more malleable attitudes. Second, heavy gamers accept in-game advertising as an inevitable part of the future of their play". Gamers even appreciate "cool" companies that advertise in games ${ }^{3}$. Third, in-game advertising is non-obtrusive. Unlike TV commercials that disrupt a running TV program, advertised brands are carefully and subtly integrated into the game. Most importantly, however, games differ from TV programs and movies in terms of their inherent interactivity. Playing games is an active process, which may even include some form of forced exposure to the advertised brand. TV commercials typically entail shallow encoding of product information. Carefully integrating brands as an important part of the storyline may result in deliberately directing the player's avatar to a billboard or even "force" the player to use a particular product (e.g., Coca Cola). From a human memory perspective, this process induces deep encoding of the product information, which, according to the levels-ofprocessing approach (Craik \& Lockhart, 1972), will serve as a superior basis for later retrieval. Taken together, in-game advertising may be among the most effective forms of advertising because of superior brand exposure in terms of frequency, duration, and intensity or depth of encoding.

\section{AdRacer Testing System}

The AdRacer system was designed as a flexible tool for adaptive and ecologically valid experimental testing that warrants continuous interaction between the player and virtual 3D game environment. The system comprises driving simulator, 3D racing game environment, and recording of gaze directions and bio signals (e.g., EEG). Additional questionnaires and post-driving memory tests complete the in-depth analysis of advertising efficiency and game effects on the player (Figure 1).

The AdRacer is equipped with a 30-inch back-projected display for immersive gaming, an adjustable car seat, stereo sound, and a steering wheel and foot pedals for realistic driving experiences. The system uses a non-obtrusive single-camera infrared eye-tracking device. Figure 2 illustrates the AdRacer hardware setup.

\footnotetext{
${ }^{1} \mathrm{http} / / /$ www.yankeegroup.com/ResearchDocument.do?id=16395, acc. 7/16/2008.

${ }^{2} \mathrm{http} / / / \mathrm{www} . \mathrm{comscore} . \mathrm{com} /$ press/release.asp?press $=861$, acc. $7 / 16 / 2008$.

${ }^{3} \mathrm{http}: / / \mathrm{www} . \mathrm{massiveincorporated.com/casestudies.html,} \mathrm{acc.} \mathrm{7/16/2008.}$
} 


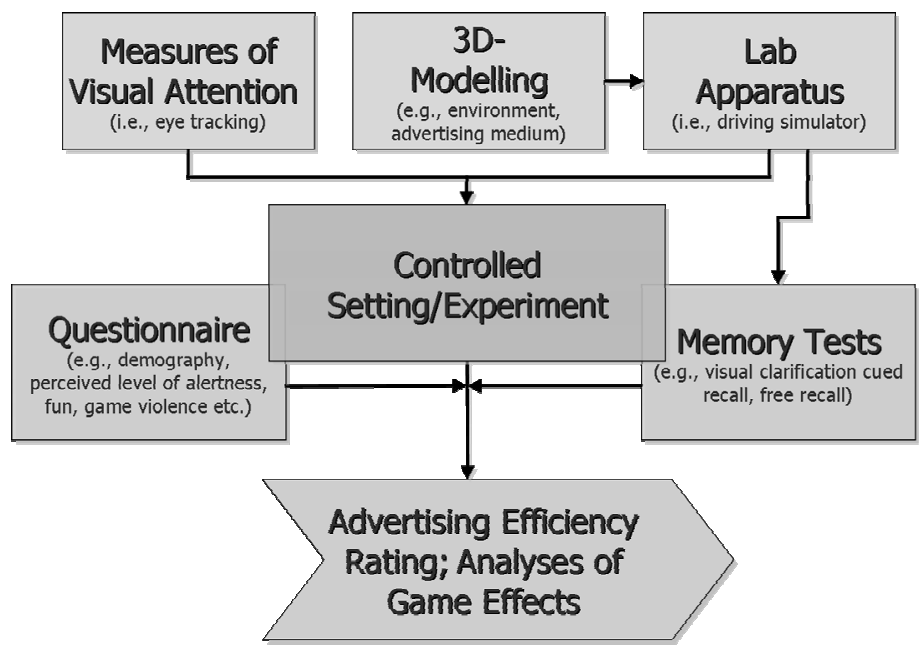

Fig. 1. The AdRacer testing system. See text for further details.
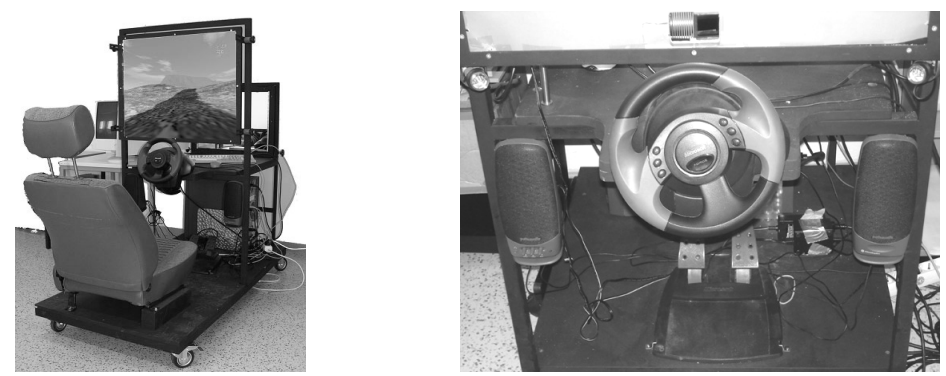

Fig. 2. The AdRacer driving simulator, together with steering wheel, stereo speaker, foot pedals, eye-tracking camera (above steering wheel) and infrared emitter (above speakers)

The virtual 3D environment in the novel AdRacer game used in the pilot study was designed using the open source TriBase game engine, which supports Microsoft's DirectX 9 graphics standard and comprises a wide range of I/O and A/V libraries (Scherfgen, 2006). We implemented different 3D models for billboards, trees, houses, rotating geometrical target shapes (i.e., rings and diamonds), and animated humanlike characters. For the violent version, two different 3D avatar "targets" were designed (a man in a business suit, and a woman in a wheelchair), both with their hands down. To render a more realistic impression, a second model was designed for each avatar with their hands held up. Once the player approached the avatars, the "handsup" model automatically replaced the "hands-down" model. This simple two-stage animation proved to be an effective method to induce the lively impression of a "real" person spontaneously reacting to a fast approaching car.

Based on different types of ground tiles (i.e., basic straight, curve-left, and curveright textures that define the course of the road), and the number and positions of bonus items and billboards, the TriBase engine generated an open race track. The race 
track is visually situated in the suburbs, with different types of houses and trees sparsely distributed. In the present study, no other cars or AI-based non-player characters appeared (except for the avatars in the violent version). The game's underlying physics engine rendered a realistic driving behavior, including screeching tires and crashes whenever participants left the road and bumped into houses or trees.

Both game versions had a first-person point of view that shifted realistically according to the player's input (Figure 3). By providing "unfiltered" sensory cues, feelings of spatial self-presence are strengthened, thus further inducing involvement and immersion in the game environment (e.g., Ijsselsteijn, 2001). The first-person point of view also plays an important role in current models of media effects. Because of adopting the acting character's role, the first-person view supports identification. In the General Aggression Model (Anderson \& Bushman, 2002), these attributes help form chronically accessible mental models, which change an individual's personality and, thus, help to explain how violent video games influence aggressive personality.
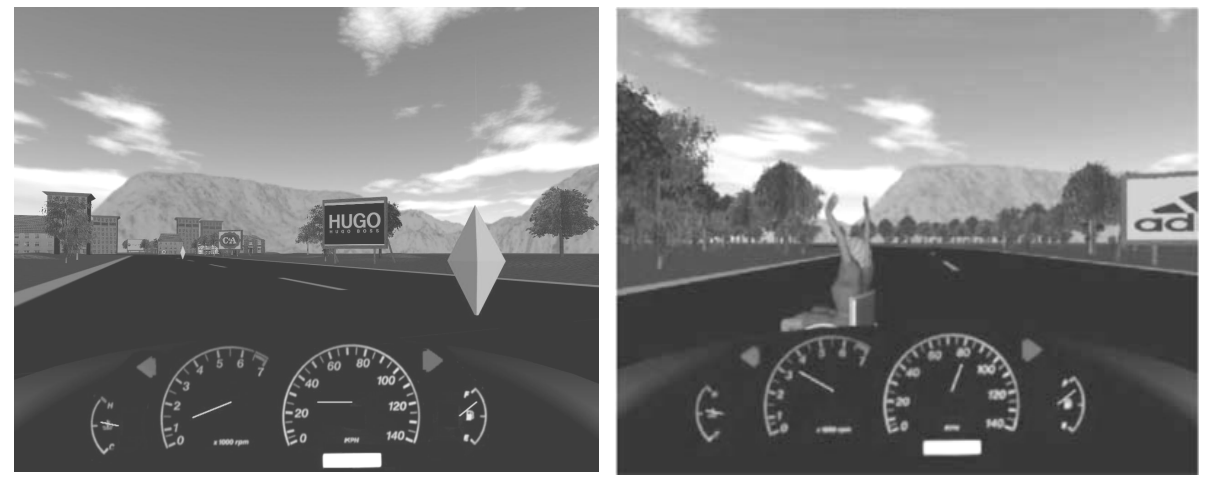

Fig. 3. Hitting items in the nonviolent version (left) and running over pedestrians in the violent version (right) of the AdRacer racing game, together with billboard ads, as seen through the imaginary windshield. An animated dashboard further increased the realistic impression.

To further understand the effects of in-game advertising, an important potential mediator of memory-participants' gaze directions-was also measured. Analyzing potential differences in visual attention towards brand logos helps to understand the mechanisms that underlie brand memory, and gaming behavior per se. Gaze directions provide a continuous measure without interrupting processing. Unlike verbal statements, gaze directions are largely automatic (i.e., resistant to strategic control). Eye movements were continuously recorded in both conditions during the driving game. The AdRacer system uses the InSight ${ }^{\mathrm{TM}}$ eye-tracking device (CSensoMotoric Instruments), which only requires a headband with a marker tracked by a single camera, thus indicating the player's head position. Based on three infrared emitters, two corneal reflexes are produced giving an estimate of the cornea's curvature. The eye tracker software provides information on head orientation, lid opening, and gaze direction. In the present study, we will confine ourselves to gaze direction. Using a nonobtrusive device further supports the ecological validity of the interaction between player and game environment. 
The AdRacer display was virtually divided into 42 areas of interest to calibrate individual gaze direction. We programmed a merging routine that combined the recorded signals into one file container, including the AdRacer game data. An additional database handled both file container entries and memory data.

\section{Pilot Study: Testing Memory for Product Information}

Typically, people watch TV commercials or play sports simulation games in the privacy of their homes, or pass by billboards on their daily way to the office, and then go shopping later. Contact with product information thus occurs prior the actual buying situation. This temporal and spatial decoupling of encoding and retrieval is also reflected in theoretical models of people's complex response to advertising in which memory represents a crucial component (Shimp \& Gresham, 1983). Memory has been repeatedly demonstrated to mediate consumer behavior (Bagozzi et al., 1992; Bushman, 2005). Brand placements, for example, are thought to increase the level of familiarity with the advertised brand so that consumers will later remember and eventually buy the product (d'Astous \& Chartier, 2000).

In addition to formal aspects (e.g., logo design), memory for advertised brands is also affected by contextual factors. For example, embedding the product information in a violent TV program context is known to impede brand memory compared to a neutral program (see Bushman \& Phillips, 2001 for a meta-analytic review). In these studies, participants watch TV with commercial breaks. They are then given surprise tests of memory. Despite comparable levels of arousal, entertainment, and involvement, participants recall fewer brands embedded in the violent TV program (e.g., "24") compared to the nonviolent program (e.g. "America's funniest animals").

In the light of the aforementioned trend towards in-game advertising, the AdRacer pilot study tested whether game violence will have detrimental effects on brand memory similar to those found with TV violence. Little is known about brand memory in games; recently, it has even been characterized "a virtually unresearched area" (Yang et al., 2006). The few studies that have already addressed memory for in-game advertising yielded only low brand memory. For example, participants in a first-person shooter recalled going past billboards, yet revealed little memory for brands (Chaney et al., 2004). To our knowledge, no study has directly compared memory for advertised brands in matching versions of a violent and a nonviolent game.

\subsection{Method}

The experimental pilot study that tested the AdRacer system addressed the effects of game context on memory for brands that appeared as billboard ads in a novel racing game-does the violent game context impair participants' memory for brands similar to TV violence? Two matching versions of the game were therefore designed that differed only in terms of violence. In the nonviolent version, participants were rewarded for running over animated geometrical shapes distributed along the race track. In the violent version, participants were rewarded for running over pedestrians (i.e., human-like avatars). Both groups were then given surprise memory tests. 


\subsubsection{Design and Participants}

A 2 (game content: violent, nonviolent) x 2 (memory test: cued recall version of a brand clarification task, free recall of brands) mixed-factorial design was used. Game content served as a between-subjects factor; participants thus either played the violent or the nonviolent version of the racing game. Memory test was varied within subjects. However, the order of the tests was fixed; participants started with the clarification task (cued recall) and then proceeded with free recall.

Participants $(N=19)$ were students and faculty members from different faculties at the University of Luebeck. In the violent condition, 2 females and 7 males (mean age $=24.40, S D=4.17$ ) participated $^{4}$. In the nonviolent condition, there were 3 females and 7 males (mean age $=22.56 S D=5.41$ ). Faculty members volunteered whereas students received course credit for their participation. Members of both groups were naïve to the experiment and its goals.

\subsubsection{Materials}

Sixty-four high quality versions of corporate brand logos from different product categories were selected from the Internet ${ }^{5}$. A pretest with 20 different participants corroborated that all logos represented known brands. For the driving game, logo size was adjusted to fit the VR version of the standard German "Mega light" billboard size $(252 \mathrm{~cm} \times 356 \mathrm{~cm})$. For the clarification task that was used as a cued recall test, brand logos were adjusted to fit into 480 (width) by 640 (height) frame size.

All participants were given the same race track in the driving session. However, 32 different logos were randomly selected for each participant. Brand logos were assigned billboard positions that had been pre-rendered by the game engine. Each logo was shown as a 2D-billboard ad and appeared three times during an individual driving session. Hence, each participant encountered a total of 96 brand logos in the study session ("learned list logos"). For the clarification cued recall test, 16 brand logos were taken from the learned list ("repeated") and were presented together with the 16 logos that had not been previously encountered in the driving session ("new") 6 . Repeated and new items were presented in random order. Only one brand logo was displayed at a time in the clarification cued recall test.

\subsubsection{Procedure}

The experiment comprised, in chronological order, the training session to accustom participants to the driving game, the study session in which eye movements were recorded while participants played the driving game and encountered billboard ads, the questionnaire session that addressed demographical factors and participants' ratings of the AdRacer game, and the memory tests.

Participants were tested individually in a computer lab. They randomly assigned themselves to either the violent or nonviolent version by drawing a slip of paper. After adjusting the driver's seat in front of the AdRacer display the experimenter

\footnotetext{
${ }^{4}$ A third female participant in the violent condition started the driving session but soon had to quit because of experiencing intense simulator sickness.

${ }^{5}$ http://www.webchantier.com/_index_en.html, acc. 7/16/08.

${ }^{6}$ The remaining 16 brand logos from the learned list were used in another memory test that yielded similar results, but will not be reported here.
} 
attached the headband and calibrated the eye tracker. To compensate for individual differences in experiences with driving games, a learning criterion required 180,000 bonus points to be scored within three minutes of driving in the training session. A special nonviolent training game version was used. Participants were told to maximize their personal bonus by hitting (i.e., passing through) items that displayed their score and appeared on the left and right lane. Hitting an item triggered a cheerful sound, a reddened screen image (flash) for $200 \mathrm{~ms}$, and the bonus score, which was shown in the middle of the screen. No billboard ads were shown during training. All participants met the criterion within three attempts.

Next, both groups were given identical instructions for the driving session (study phase); they were told to maximize their personal bonus to make it into the high score list. To further increase motivation, participants were told that the top 3 scorers would receive additional gifts. Billboard ads were not mentioned, nor were participants in the violent condition told that avatars would now replace neutral items. Driving session was self-paced and ended when participants passed by all 96 brand logos.

In the violent condition, "running over" an avatar immediately triggered screams of pain either from a female or male voice depending on the gender of the character. Also, a splashy sound was played and the windshield was covered with blood stains for $200 \mathrm{~ms}$. As was true for the nonviolent version, participants were immediately rewarded with the bonus score.

Following the driving session, participants received the questionnaire. Next, they started the first memory test. They were told that some of the hidden brand logos in the upcoming visual clarification test had been presented earlier on billboard ads. Therefore, they should deliberately recall the driving session. Brands were presented one at a time. Each trial started with a verbal cue displayed for $1,500 \mathrm{~ms}$, immediately followed by a fixation cross in the middle of the computer monitor. After $500 \mathrm{~ms}$, the fixation cross disappeared and the screen was blackened for $1,000 \mathrm{~ms}$. Then, the brand clarification started automatically. The computer program started time recording as soon as a visually degraded brand appeared on the screen. Visual masking was automatically evenly reduced (i.e., gradually clarified) by randomly removing blurring pixels at a 2 percent per second rate, starting with $100 \%$ noise blur. Participants were instructed to immediately stop the clarification process (and, thus, recording of reaction time) by pressing the Space bar when they visually identified the brand. The program immediately replaced the degraded logo with the instruction to type in the name of the recognized brand. Participants accustomed themselves to the task on five training trials (using new brands) and then completed 32 test trials.

In the concluding free recall test, participants were given a blank form. They were told to type in only brand names they remembered from the driving session. Finally, participants were debriefed. The entire experiment took 50 to 60 minutes.

\subsection{Results}

Findings from the questionnaire will be described first (4.2.1), followed by memory data (4.2.2). We will also address whether visual attention (gaze directions) affected brand memory (4.2.3). An exhaustive description of all results would go beyond the scope of this paper. Hence, we will confine ourselves to the most important findings. 


\subsubsection{Questionnaire}

The 20 items of the questionnaire addressed demographical factors, like age, gender, occupation, computer and gaming expertise, and personal frequency of gaming, which are known to moderate measures of gaming behavior. Lower ratings on the 4-point scale indicated stronger affirmation. Two-sided $t$-tests $(\alpha=.05)$ revealed only one significant effect: game versions substantially differed in violence, $t(17)=6.08, p<.01$. As expected, participants in the nonviolent condition $(M=3.67, S D=0.50)$ did not find the game violent at all, whereas players of the violent version $(M=1.80, S D=0.79)$ confirmed that they had played a violent game. No other comparison was significant ( $p \mathrm{~s} \geq .11$ ), indicating that game versions were matching in terms of handling, perceived realism, and subjective estimations of arousal, excitement, alertness, and fun.

\subsubsection{Memory Data}

\section{Cued Recall (Clarification Task)}

The number of erroneously recalled brand names was below 3\%. Therefore errors were not calculated separately. First, we compared baseline performance, that is, group means for brand logos that had not been encountered in the study phase. The one-factorial ANOVA with game content serving as a between-subjects variable revealed no group difference, $F(1,17)<1$. Not surprisingly, participants playing the violent version $(M=13,276 \mathrm{~ms}, S D=3,006)$, and the nonviolent version $(M=13,548 \mathrm{~ms}$, $S D=3,105)$ did not differ in terms of the time it took them to identify blurred brand logos that had not been shown earlier. Next, we compared group means for brand logos that were repeated from the study phase. Contrary to our hypothesis, the onefactorial ANOVA revealed no group difference, $F(1,17)<1$. Participants playing the violent version $(M=12,878 \mathrm{~ms}, S D=2,894)$ were even numerically faster than their colleagues in the nonviolent version $(M=13,614 \mathrm{~ms}, S D=2,923)$.

\section{Free Recall}

There was no substantial group difference in the free recall test, $t(17)=-.095, p=.93$. In the nonviolent condition, participants recalled $9.03 \%(M=2.89, S D=2.09)$ of the brand logos encountered in the driving session. Participants playing the violent version recalled $9.38 \%(M=3.00 S D=2.91)$.

\subsubsection{Eye-Tracking Data}

Due to technical problems with the eye-tracking device, data from only 5 participants in the nonviolent and 8 participants in the violent condition were obtained. Because of the low number of observations, only descriptive statistics will be presented. First, the mean number of fixations (hits) for billboard ads was calculated (Table 1, upper half). Recordings from gaze directions up to $3 \%$ displacement from the outer billboard frame were accepted as hits ${ }^{7}$. Please note that each of the 32 different brand logos was repeated three times during an individual driving session.

Apparently, repeating brand logos as billboard ads in the driving session positively affected participants' gaze directions only in the nonviolent game version. In the violent version, visual contacts with billboards occurred only at chance level, irrespective of their number of appearance.

\footnotetext{
${ }^{7} \mathrm{We}$ also tested different displacement criteria $(0 \%, 1 \%$, and $5 \%)$, but obtained similar results.
} 
Table 1. Mean number of eye fixations (in \%, upper half) and mean duration of eye fixations (in $\mathrm{ms}$, lower half) in the two game versions. Standard deviations are given in parentheses.

\begin{tabular}{llrrr}
\hline & & \multicolumn{3}{c}{ Number of Appearances } \\
& & \multicolumn{1}{c}{ First } & \multicolumn{1}{c}{ Second } & \multicolumn{1}{c}{ Third } \\
\hline Number of Hits & Nonviolent & $42.50(36.28)$ & $56.25(26.88)$ & $68.75(12.50)$ \\
& Violent & $56.25(21.65)$ & $51.56(26.67)$ & $52.34(35.82)$ \\
Fixation Times & Nonviolent & $286(268)$ & $234(323)$ & $276(320)$ \\
& Violent & $166(248)$ & $192(265)$ & $167(263)$ \\
\hline
\end{tabular}

Next, mean fixation time for "hits" was calculated (Table 1, lower half). Playing the violent game version not only resulted in numerically fewer, but also shorter eye contact with billboard ads. Compared to the nonviolent version, thus, "violent" gamers generally seem to pay less visual attention to ads. In addition, the expected correlation between fixation times and number of hits was significant in this condition, $r=.82, p=.01$, but not in the nonviolent version, $r=-.22, p=.73$.

In a final step, eye-tracking data and memory results were compared. To our surprise, there was no significant correlation between gaze direction and memory performance for repeated brands in the later cued recall (clarification task). In the nonviolent game version, additional ( $r=-.24)$ and longer eye contact $(r=-.35)$ with billboard ads only numerically speeded later identification of repeated brands. In the violent condition, however, the positive yet insignificant correlation of number of hits $(r=.22)$, and fixation time $(r=.39)$, respectively, indicates that eye contact with the brands even numerically slowed down brand identification.

\subsection{Discussion}

The AdRacer pilot study yielded surprising findings. Results from both memory tests sharply contrast with our hypothesis based on previous findings that demonstrated the detrimental results of TV violence on memory (e.g., Bushman \& Phillips, 2001) ${ }^{8}$. In our study, game violence (i.e., running over pedestrians in a racing game) did not at all impede memory for brands previously shown as billboards. We found no significant differences on either memory test between violent game players and nonviolent game players. Visual attention did not mediate the results either. How do we make sense of these findings?

Most importantly, only a small number of participants were tested in the AdRacer pilot study. Technical problems with the eye-tracking device further reduced the number of observations. First and foremost, we thus have to replicate our findings with a larger sample to increase the reliability of our findings.

\footnotetext{
${ }^{8}$ In the literature on media effects on brand memory, two studies have reported a similar result (i.e., greater memory performance in the violent condition). The authors of the first study did not provide a cogent explanation for their findings (Droulers \& Roullet, 2004). The second study showed that reinstating a "violent mood" at test might support retrieval of violent commercials (Gunter et al., 2005). In our study, however, brands were emotionally neutral and memory was tested with an emotionally neutral visual clarification task and free recall.
} 
In addition, one could argue that game versions did not differ in level of violence they conveyed. As expected, however, participants' ratings confirmed that collecting geometrical shapes and running over pedestrians significantly differed in terms of violence. Although subjective ratings do not fully rule out the possibility that versions might have been too similar-we did not record objective physiological data to indicate typical patterns of bodily reaction to violence, for example-we are confident that we have successfully varied game violence in the present study.

The detrimental effect of TV violence on brand memory has been attributed to violence drawing visual attention away from the product information to the program itself, thus preventing brand memory traces from being established (i.e., attentiongrabbing effect; Bushman \& Bonacci, 2002; Gunter et al., 2005). This thesis is also supported by recent models on the effects of emotional arousal on memory binding (Mather, 2007). Attention is assumed to be primarily paid to the emotionally arousing object. At the same time, contextual features are neglected and therefore not properly encoded. In our study, we expected participants playing the violent racing game to pay attention to the task, and, thus, to their potential victims (i.e., the emotionally arousing objects), but not to task irrelevant contextual features like billboards. Interestingly, "violent players" in fact dominated the high score list in the driving session; six of the top ten players (including ranks 1 and 2) played the violent version with a mean score of 1,212.500 (mean score for "nonviolent players" was 1,199.875). Likewise, eye-tracking data indicated that participants in the violent version did not benefit from repeating brand logos. Apparently, participants closely did pay attention to the driving task.

But if playing the violent version indeed meant paying close attention to violent acts, but not to brand logos, why did participants in the nonviolent condition perform so poorly in the memory tests? One attempt we made to match both conditions as closely as possible could have backfired on the study, selectively affecting the nonviolent version. To compensate for the blood-covered windshield in the violent condition, passing through a geometrical shape triggered a flash of red light for $200 \mathrm{~ms}$. In contrast to the semi-transparent blood splashes, the red flash was opaque and entirely covered the screen. Hence, a masking effect may have disrupted visual perception in the nonviolent condition. Visual masking is an effective way to erase iconic memory, thus preventing consolidation of representations (Enns \& Di Lollo, 2000). After the mask disappeared, participants in the nonviolent condition may have "re-fixated" by means of additional eye movements. This post-hoc explanation would fit both memory and eye-tracking data (i.e., additional fixations and greater fixation time) in this condition and could easily be tested in the next study.

\section{Concluding Remarks}

The present paper introduced the AdRacer system for systematic analyses of game effects. AdRacer offers high levels of experimental control in a realistic and immersive 3D game environment. Because of the system's underlying architecture, gaming information may also be utilized in subsequent tests of cognitive processes (e.g., memory). By integrating additional methods of detecting the player's gaze direction and physiological data (e.g., EEG), AdRacer also supports the in-depth analysis of 
actual gaming behavior. Further analyzing the processes engaged during game play is an indispensable prerequisite of understanding the effects or consequences of game violence on players' reactions to brands.

AdRacer was used in a pilot study that tested the effects of game violence on brand memory. Remembering product information is becoming increasingly important in the upcoming economic efforts of in-game advertising, yet little is known about its effects (Yang et al., 2006). The overall low number of participants certainly limits the reliability of our findings. However, recordings of gaze directions and analyses of brand memory revealed two remarkable results. First, encountering task irrelevant brand information may establish memory traces that are later reenacted. This is an important finding for advertisers thinking of product placement in computer and video games. However, advertisers should also note that this memory effect was far from being irreversible. Rather, memory was sensitive to contextual factors. At this point, we cannot specify whether this sensitivity was due to game version (violent or nonviolent), or short visual flashes. Future studies will further our understanding if game violence will mirror the detrimental effect of TV violence on brand memory, which has been repeatedly demonstrated (e.g., Bushman \& Phillips, 2001). Given the increasing economic relevance, the important role of memory processes in the context of advertising, and the societal implications of violent games, future studies are badly needed. The AdRacer provides an excellent platform for conducting this research.

\section{Acknowledgments}

The authors wish to thank Olaf Christ, Sebastian Otto, Henning Hansen, and Gregor Radzimski for their pivotal programming work and running the experiments.

\section{References}

1. Anderson, C.A., Bushman, B.J.: Human aggression. Annual Review of Psychology 53, 27-51 (2002)

2. Anderson, C.A., Bushman, B.J.: Effects of violent video games on aggressive behavior, aggressive cognition, aggressive affect, physiological arousal, and prosocial behavior: a meta-analytic review of the scientific literature. Psychological Science 12(5), 353-359 (2001)

3. Bagozzi, R.P., Baumgartner, H., Yi, Y.: State versus action orientation and the theory of reasoned action: An application to coupon usage. Journal of Consumer Research 18, 505518 (1992)

4. Bushman, B.J.: Violence and sex in television programs do not sell products in advertisements. Psychological Science 16(9), 702-708 (2005)

5. Bushman, B.J., Bonacci, A.M.: Violence and sex impair memory for television ads. Journal of Applied Psychology 87(3), 557-564 (2002)

6. Bushman, B.J., Phillips, C.M.: If the television program bleeds, memory for the advertisement recedes. Current Directions in Psychological Science 10, 44-47 (2001)

7. Chaney, I.M., Ku-Ho, L., Chaney, J.: The effect of billboards within the gaming environment. Journal of Interactive Media 5(1), 54-69 (2004) 
8. Craik, F.I.M., Lockhart, R.S.: Levels of processing: A framework for memory research. Journal of Verbal Learning and Verbal Behavior 11, 671-684 (1972)

9. d'Astous, A., Chartier, F.: A study of factors affecting consumer evaluations and memory of product placements in movies. Journal of Current Issues and Research in Advertising 22(2), 31-40 (2000)

10. Droulers, O., Roullet, B.: Does crime pay for violent program-embedded ads? Advances in Consumer Research 31, 1-6 (2004)

11. Enns, J.T., Di Lollo, V.: What's new in visual masking? Trends in Cognitive Science 4, 345-352 (2000)

12. Ijsselsteijn, W.A., Freeman, J., De Ridder, H.: Presence: Where are we? CyperPsychology \& Behavior 4(2), 179-182 (2001)

13. Gunter, B., Furnham, A., Pappa, E.: Effects of television violence on memory for violent and nonviolent advertising. Journal of Applied Social Psychology 35(8), 1680-1697 (2005)

14. Mather, M.: Emotional arousal and memory binding. An object-based framework. Perspectives on Psychological Science 2(1), 33-52 (2007)

15. von Salisch, M., Oppl, C., Kristen, A.: What attracts children? In: Vorderer, P., Bryant, J. (eds.) Playing Video Games: Motives, Responses, and Consequences, pp. 147-163. Erlbaum, Mahwah (2006)

16. Scherfgen, D.: 3D-Spieleprogrammierung mit DirectX 9 und C++. Hanser, München (2006)

17. Shimp, T.A., Gresham, L.G.: An information processing perspective of recent advertising. Current Issues and Research in Advertising 9, 36-79 (1983)

18. Yang, M., Roskos-Ewoldsen, D.R., Dinu, L., Arpan, L.M.: The effectiveness of "in-game" advertising. Comparing college students' explicit and implicit memory for brand names. Journal of Advertising 35(4), 143-152 (2006) 